\title{
PAPER
}

Cite this: Nanoscale, 2013, 5, 4776

\section{Dimethylformamide-mediated synthesis of water-soluble platinum nanodendrites for ethanol oxidation electrocatalysis $\dagger$}

\author{
Stefanos Mourdikoudis, ${ }^{\text {a }}$ Mariana Chirea, ${ }^{\mathrm{b}}$ Thomas Altantzis, ${ }^{\mathrm{c}}$ Isabel Pastoriza- \\ Santos, ${ }^{a}$ Jorge Pérez-Juste, ${ }^{a}$ Fernando Silva, ${ }^{b}$ Sara Bals ${ }^{c}$ and Luis M. Liz-Marzán ${ }^{\star}$ ade \\ Herein we describe the synthesis of water-soluble platinum nanodendrites in dimethylformamide (DMF), \\ in the presence of polyethyleneimine (PEI) as a stabilizing agent. The average size of the dendrites is in \\ the range of 20-25 $\mathrm{nm}$ while their porosity can be tuned by modifying the concentration of the metal \\ precursor. Electron tomography revealed different crystalline orientations of nanocrystallites in the \\ nanodendrites and allowed a better understanding of their peculiar branching and porosity. The high \\ surface area of the dendrites (up to $22 \mathrm{~m}^{2} \mathrm{~g}^{-1}$ ) was confirmed by BET measurements, while X-ray \\ diffraction confirmed the abundance of high-index facets in the face-centered-cubic crystal structure of \\ Pt. The prepared nanodendrites exhibit excellent performance in the electrocatalytic oxidation of \\ ethanol in alkaline solution. Sensing, selectivity, cycleability and great tolerance toward poisoning were \\ demonstrated by cyclic voltammetry measurements.
}

Received 21st February 2013 Accepted 28th March 2013

DOI: $10.1039 / c 3 n r 00924 f$

www.rsc.org/nanoscale fuel cells to catalyze the fuel oxidation and the oxygen reduction reactions. $^{3}$

The fuel cell technology faces certain problems toward its commercialization. There are issues concerning the reliability of both anode and cathode catalysts. In particular, platinum catalysts are readily poisoned by contaminants (e.g. CO) during the oxidation of fuels. Concerning the type of fuel cells, numerous studies have been implemented on direct alcohol fuel cells, which are known as an interesting power source for portable electronic devices and on-board vehicles. In comparison with methanol, ethanol is more attractive thanks to its higher energy density, higher boiling point and lower toxicity. In addition, ethanol can be massively produced from agricultural products or biomass. Nevertheless, the achievement of the commercialization of direct ethanol fuel cells (DEFCs) shall not be realized unless the activity and durability of the electrocatalysts are improved. Pt-based electrocatalysts have not drawn significant interest so far for ethanol oxidation, probably because of their slower kinetics - compared to Pd - and lower activity. ${ }^{4}$

Dendrite, star-shaped, flower-like and other anisotropic morphologies of Pt nanostructures are interesting in terms of potential catalytic applications because they contain surfaces with high-index crystal facets, which allow a relative abundance of active corner and edge sites. ${ }^{5}$ Such branched morphologies typically possess a high surface area, which in general helps to increase the catalytic activity of nanoscale structures. However, it is generally difficult to produce highly anisotropic Pt nanostructures, mainly because $\mathrm{Pt}$ has an inherently highly symmetric face-centered cubic (fcc) crystal lattice. ${ }^{6}$ For this reason, Pt nanocrystals do not have any intrinsic driving force to 
grow into such highly anisotropic shapes, making the solutionphase synthesis of branched morphologies quite challenging. Despite this, some reports are available on the chemical synthesis of Pt nanostructures with such peculiar morphologies, obtained for example by organometallic or polyol pathways. $^{7}$

Our group has reported the synthesis of highly catalytic single-crystal dendritic Pt nanostructures supported on carbon nanotubes, based on a combined reduction of $\mathrm{K}_{2} \mathrm{PtCl}_{4}$ by citrate and borohydride ions. ${ }^{8}$ In the current work we present an improved synthesis of platinum nanodendrites via the thermolytic reduction of a different platinum precursor, $\mathrm{Pt}(\mathrm{acac})_{2}$, in DMF. This protocol can be easily scaled up for the production of larger quantities, additionally allowing one to tune the porosity of the nanoparticles by simply varying the concentration of the precursor. We used branched polyethyleneimine, PEI, not only as a stabilizer but also to assist the reduction process driven by DMF. PEI is a hydrophilic polymer with primary, secondary and tertiary amino groups and an overall positive charge in neutral aqueous solution. Due to its high positive charge, PEI is widely used as a NP stabilizer to achieve specific surface functionalization. PEI is particularly attractive when catalytic applications are aimed at, because rather than blocking active sites, as reported for other polymers such as polyvinylpyrrolidone (PVP), ${ }^{9}$ PEI can even enhance electrochemical reactions. ${ }^{10}$ For example, Yi et al. have recently shown that PEI-functionalized Pt-carbon nanotube composite materials show better tolerance to carbon monoxide and higher degree of oxidation of methanol to $\mathrm{CO}_{2}$ than their PVP-functionalized counterparts. ${ }^{11}$

We have explored several reaction protocols toward the formation of $\mathrm{Pt}$ nanodendrites via PEI-assisted chemical reduction. Apart from standard characterization techniques such as XRD (X-ray diffraction) and TEM, electron tomography was also used to avoid ambiguous interpretations that usually result from 2D-TEM imaging. We additionally present and discuss the remarkable electrocatalytic properties of these $\mathrm{Pt}$ nanodendrites for ethanol oxidation.

\section{Results and discussion}

\subsection{Synthesis and characterization}

The thermolytic reduction of $\mathrm{Pt}(\mathrm{acac})_{2}$ at $154^{\circ} \mathrm{C}$ in $\mathrm{DMF}$, in the presence of PEI (Table 1), yields platinum nanostructures with dendritic morphology (Fig. 1). These nanodendrites are readily dispersible in water to form solutions with prolonged colloidal stability ( $\zeta$-potential $\sim+44 \mathrm{mV}$ ). Varying the concentration of platinum acetylacetonate, while keeping all the other reaction parameters unchanged, leads to modifications in the porosity of the dendrites. More specifically, we used three different precursor concentrations (3 $\mathrm{mM}, 15 \mathrm{mM}$ and $30 \mathrm{mM}$, for samples Pt1-PEI, Pt2-PEI and Pt3-PEI, respectively - see Experimental part for details). Nanoparticles in sample Pt1-PEI display a rather 'compact' shape and an average size of $\sim 25.4 \pm$ $3.3 \mathrm{~nm}$ (Fig. 1a and S1a-a' in ESI $\dagger$ ). When the precursor concentration was increased, nanodendrites with slightly smaller sizes were obtained, which showed a tendency to form more porous (less compact) structures (Fig. 1b and S1b-b' (ESI $\dagger$ ) for sample Pt2-PEI, Fig. 1c and S1c-c' $\dagger$ for sample Pt3-PEI). The measured size for sample Pt2-PEI was $22.3 \pm 3.2 \mathrm{~nm}$, while sample Pt3-PEI had a size of $21.1 \pm 3.5 \mathrm{~nm}$. The different porosity is also reflected in the respective values of specific surface area. Indeed, the BET measurements yielded surface area values of $10 \mathrm{~m}^{2} \mathrm{~g}^{-1}$ for sample Pt2-PEI, but $22 \mathrm{~m}^{2} \mathrm{~g}^{-1}$ for sample Pt3-PEI.

Dendrite formation was monitored by extracting aliquots at different reaction times for TEM analysis. Thus, after $20 \mathrm{~min}$ of reflux time, both small dendrites and 'oligopods' with an average size of $\sim 16.5 \mathrm{~nm}$ were observed (Fig. S2 in ESI $\dagger$ ), while nanodendrites were completely formed after $1 \mathrm{~h}$ of reaction, and no further growth was observed even after prolonged heating for $24 \mathrm{~h}$. Interestingly, DMF was able to reduce $\mathrm{Pt}(\mathrm{a}$ cac $)_{2}$, even at a relatively moderate temperature, $154{ }^{\circ} \mathrm{C}$, whereas the same precursor could not be reduced at $180^{\circ} \mathrm{C}$ by milder reductants such as oleylamine. ${ }^{12}$ It has been suggested that DMF can also serve as a temporary protective agent for Pt nanocrystals through the interaction between the carbonyl amide group and $\mathrm{Pt}^{13}$ However, in the synthetic protocols applied in the current study, such a protective effect of DMF was not observed, since significant aggregation was observed in the absence of PEI. Moreover, apart from the stabilizing role of PEI, its co-reducing effect was confirmed by running the same reaction with PEI dissolved in an 'inert' solvent such as anisole at $154{ }^{\circ} \mathrm{C}$ and finding that platinum acetylacetonate was still reduced, however the obtained particles had a different shape than with DMF (not shown here). The reducing ability of PEI has been previously reported for the preparation of $\mathrm{Au}$ nanoparticles. ${ }^{\mathbf{1 4}}$

Table 1 Overview of the reaction parameters for all platinum samples presented in this study (PEI concentration $=600^{\mathrm{a}} \mathrm{mM}$ for all samples, $T=154{ }^{\circ} \mathrm{C}, t=24 \mathrm{~h}, \mathrm{DMF}$ volume $=30 \mathrm{~mL}, \zeta$-potential of the products $\sim+44 \mathrm{mV}$ )

\begin{tabular}{|c|c|c|c|c|c|c|c|}
\hline Sample & $\begin{array}{l}\text { Precursor: } \\
\mathrm{Pt}(\text { acac })_{2}\end{array}$ & Size (nm) & $\begin{array}{l}\text { Nanodendrites } \\
\text { morphology }\end{array}$ & Solidity $\%^{b}$ & Surface area (BET) & $\begin{array}{l}\text { Current density } \\
\left(\mathrm{mA} \mathrm{cm}^{-2}\right)\end{array}$ & $j_{\mathrm{f}_{1}} / j_{\mathrm{b}_{1}}, j_{\mathrm{f}_{2}} / j_{\mathrm{b}_{2}}{ }^{c}$ \\
\hline Pt1-PEI & $3 \mathrm{mM}$ & $25.4 \pm 3.3$ & Compact & 33.0 & Not available & 36.0 & $10.3,2.3$ \\
\hline Pt3-PEI & $30 \mathrm{mM}$ & $21.1 \pm 3.5$ & 'Porous' & 22.6 & $22 \mathrm{~m}^{2} \mathrm{~g}^{-1}$ & 197.0 & $7.8,2.0$ \\
\hline
\end{tabular}

${ }^{a}$ PEI concentration in terms of monomer units. ${ }^{b}$ Solidity refers to the volume percent that is occupied by Pt to the total volume of the $3 \mathrm{D}$ electron tomography study. It is the antonym of porosity, i.e. the percentage of compactness. ${ }^{c}$ Ratios of forward to backward anodic peak current density $\left(j_{\mathrm{f}} / j_{\mathrm{b}}\right)$. 

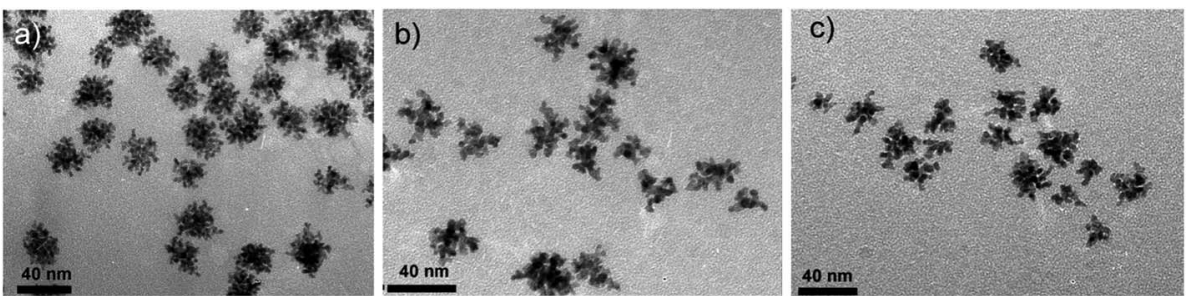

Fig. 1 TEM images for samples Pt1-PEI (a), Pt2-PEI (b) and Pt3-PEI (c).

Concerning the selection of the metal precursor, the acetylacetonate ion in $\mathrm{Pt}(\mathrm{acac})_{2}$ is a well-known bidentate chelator and has some ability to protect nanoparticles from agglomeration. ${ }^{15}$ The acac anion can react with metal ions in the presence of a base, which assists the removal of a proton from acac and shifts the equilibrium in favor of the complexation. The acac salt has been reported to give rise to more monodisperse nanoparticles than the corresponding nitrate or chloride salts. It is likely that the carbon monoxide molecules generated in situ from the decomposition of the acac ligand plays a key role in controlling the particle growth process. ${ }^{16}$

The combined effect of these reagents for nanodendrite formation was evidenced by additional experiments. Not only the use of different solvents, but also the replacement of the Pt precursor resulted in completely different morphologies in the final product (data not shown). Thus, although the particular reagents used here are well-known among synthetic chemists, the combination described in the current manuscript constitutes a novel protocol leading to such interesting anisotropic nanomaterials. In contrast to the hot-injection method, we employed the 'heat-up' approach, which can be easily scaled up, which is important toward future industrial applications. ${ }^{17}$

$\mathrm{X}$-ray diffraction analysis. In a typical example, the XRD spectrum of sample Pt3-PEI is displayed in Fig. 2. The peaks at $39.9,46.5,67.8$ and $81.6^{\circ}$ can be easily assigned to the (111), (200), (220) and (311) lattice planes of fcc-Pt (JCPDS card no. 04-0850), showing a high degree of metallic character and

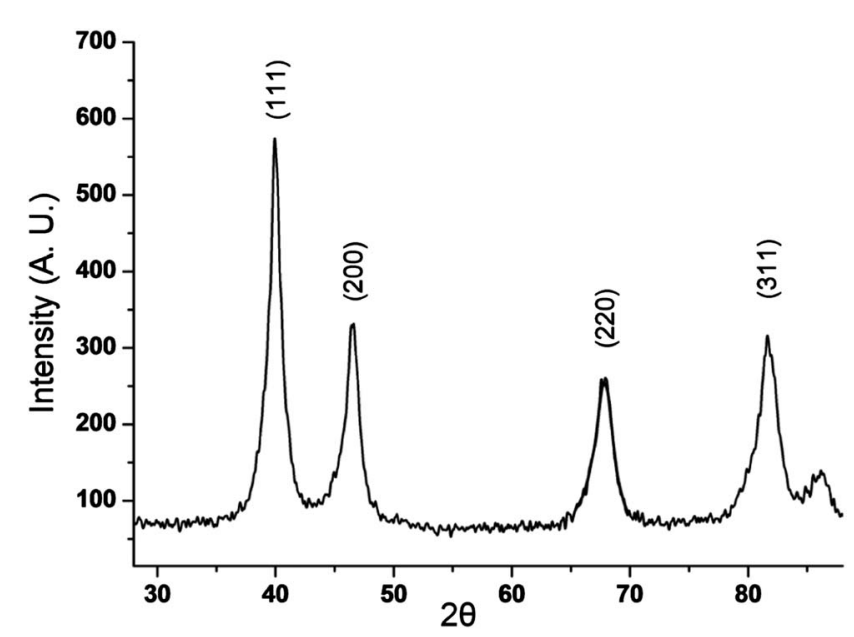

Fig. 2 XRD pattern for sample Pt3-PEI crystallinity for our nanomaterials. Similar results were obtained for the 'less-porous' dendrite samples Pt1-PEI and Pt2PEI (Fig. S3 and S4 in ESI respectively $\dagger$ ). Nevertheless, it is interesting to note that, especially for sample Pt3-PEI, while the relative intensity of the (200) peak, calculated from the peak area using (111) as a reference, is very close to the 'bulk' value (51\% vs. 53\%), the (220) and (311) relative intensities are considerably higher than the literature values. In particular, the relative intensity of the (220) peak is $39 \%$, compared to the $31 \%$ derived from the JCPDS data. Regarding the (311) peak, the observed difference is much higher, as we observe a relative intensity of $48 \%$ versus the $33 \%$ of the standard bulk values. This result indicates that the nanodendrites are rich in highindex $\{220\}$ and $\{311\}$ facets, rendering them of high interest for catalytic applications. ${ }^{18}$ Regarding the 'less-porous' samples Pt1-PEI and Pt2-PEI, the (220) and (311) peaks present the 'expected' intensities, indicating that these samples may have a lower density of catalytically active sites, or, in other words, a lower electrocatalytic activity as compared to sample Pt3-PEI. ${ }^{19}$

TEM. Advanced transmission electron microscopy techniques such as aberration corrected high angle annular dark field scanning transmission electron microscopy (HAADFSTEM) and electron tomography helped us to examine in more detail the crystalline structure and the morphology of the particles. Using HAADF-STEM, a fine electron probe scans across an area of interest and an annular detector only collects electrons that have been scattered to high angles ( $>60 \mathrm{mrad}$ ) with respect to the optical axis. This results in images where the intensity scales with thickness as well as with the square of the atomic number $Z$ of the elements present in the sample. ${ }^{20} \mathrm{High}$ resolution HAADF-STEM images for samples Pt1-PEI, Pt2-PEI and Pt3-PEI are shown in Fig. 3 (see also Fig. S5-S7 in ESI $\dagger$ ). Although there is a preferential direction in the crystalline lattice at different parts of the particles, as previously reported for other dendritic nanoparticles, ${ }^{\mathbf{8 , 2 1 b}}$ we found that several branches are oriented in different crystallographic directions, thus reflecting some degree of polycrystallinity. The contrast between the core and the peripheral branches illustrates their three-dimensional nature. Moreover, sample Pt1-PEI looks quite compact, whereas samples Pt2-PEI and especially Pt3-PEI seem to be more porous.

In all three samples we notice that each particle can be described as a dendritic entity with interconnected arms branching in various orientations. Despite that individual crystallite regions seem to be monocrystalline, the overall nanodendrite has a multiple-crystalline nature. ${ }^{22}$ Concerning 

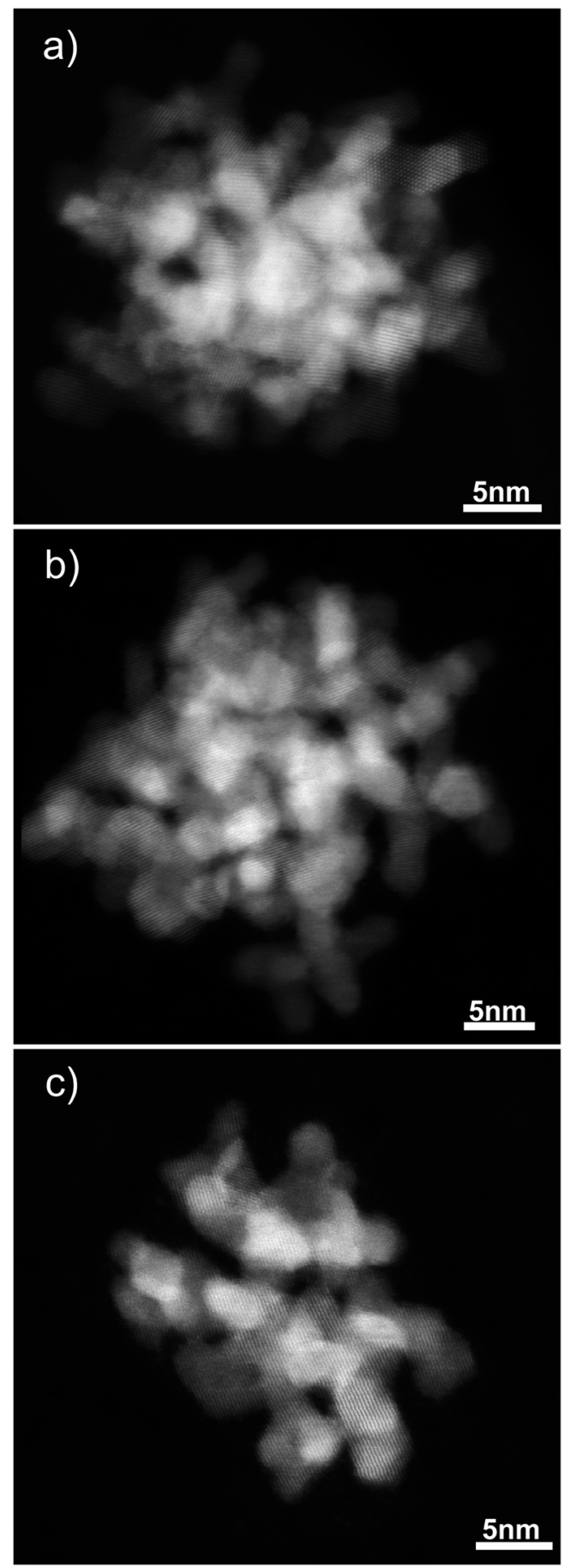

Fig. 3 High resolution HAADF-STEM images for Pt nanodendrite samples: Pt1PEI (a), Pt2-PEI (b) and Pt3-PEI (c).

the formation mechanism of our nanodendrites, it has been reported that such morphology is more likely to be produced via 'aggregation-based' nanocrystal growth (also known as the 'oriented attachment' mechanism). In the absence of strong interparticle interactions or a templating effect provided by selfassembled molecules, the initially formed particles tend to aggregate in a diffusion-controlled manner and accordingly dendritic growth can occur. In the oriented attachment process, adjacent particles self-organize so that they share a common crystallographic orientation (for example, along a given branch of the dendrite, which is also the case for our samples). However, the polycrystalline nature of the dendrites can be attributed to twinning and/or imperfectly oriented attachment characterized by a small misorientation at the interface. ${ }^{6 a, 21}$

Since all the above-mentioned images are 2D projections of $3 \mathrm{D}$ objects, electron tomography was used to acquire a more realistic 3D representation of the morphology of the $\mathrm{Pt}$ dendrites. Such a 3D reconstruction was carried out from a tilt series of 2D projection images. Aiming to confirm the apparent porosity differences between the dendrite samples, a quantification step was also performed. We calculated from the reconstructions (Fig. 4) the volume fraction that is occupied by the dendrites, by applying the same threshold for all three reconstructions. We show in Fig. 4 the volume rendering of a reconstructed Pt dendrite from each sample, at different view angles. For sample Pt1-PEI, the Pt volume fraction is $33.0 \%$, whereas for sample Pt2-PEI (Fig. 4b) it was estimated as $\sim 30.3 \%$. For Pt3-PEI, the calculated volume fraction was $22.6 \%$, revealing a clearly less compact or 'more porous' morphology. A representative 3D-rendering video for sample Pt1-PEI is provided in the ESI (ESI, $\uparrow$ see the attached video file).
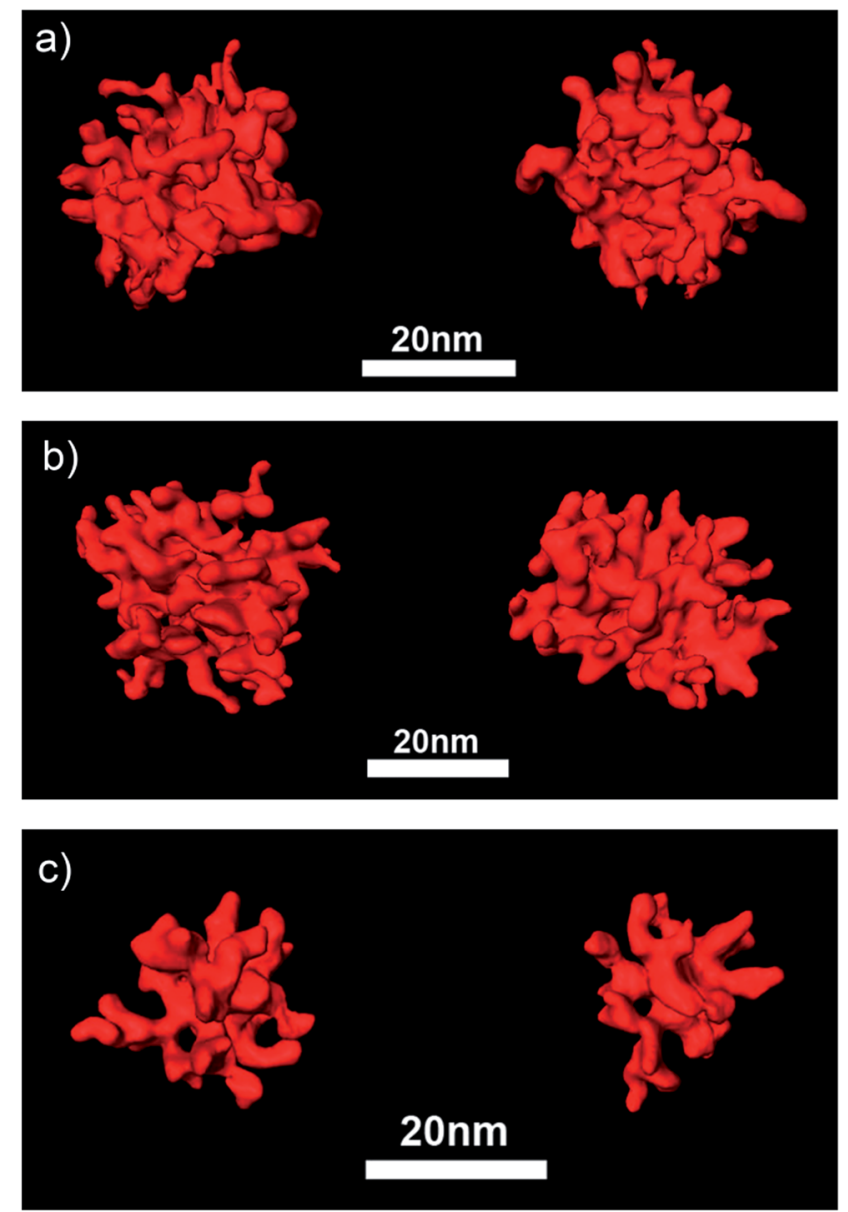

Fig. 43 D visualizations of tomographic reconstructions along different views, for Pt nanodendrite samples: Pt1-PEI (a), Pt2-PEI (b) and Pt3-PEI (c). 
The electron microscopy investigation of the dendrites' porosity and its dependence on the precursor concentration is in good agreement with XRD measurements, where the more 'porous' sample Pt3-PEI seems to have a larger population of high-index facets. In addition, as shown before, the latter sample has the highest value of surface area among all three samples $\left(22 \mathrm{~m}^{2} \mathrm{~g}^{-1}\right)$. However, in all three samples the (111) peak is much stronger than the (200) peak, a typical result for a three-dimensional randomly oriented assembly. ${ }^{23}$ The effect of precursor concentration on the growth reaction kinetics and therefore on the final morphology of the particles has also been investigated by other researchers for porous Pt nano-

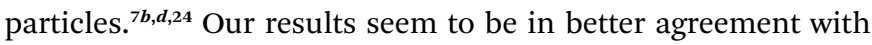
those published by Tilley's group, where a low precursor concentration (also platinum acetylacetonate in that case) was associated with the production of 'compact' morphologies, whereas a high precursor concentration yielded more porous structures. ${ }^{25}$ It is possible that a higher precursor concentration is associated with a faster nucleation, resulting in a higher population of nuclei, which would aggregate into a more open structure. On the other hand, a lower precursor concentration can lead to a 'limited' number of nuclei, which can grow into more compact structures with relatively larger sizes.

\subsection{Electrocatalytic application for ethanol oxidation}

Study of ethanol oxidation in alkaline solution at Pt-PEI modified glassy carbon (GC-Pt-PEI) electrodes. Cyclic voltammetry measurements (Fig. 5) performed at the GC-Pt1-PEI (black curve), GC-Pt2-PEI (red curve) and GC-Pt3-PEI (blue curve) electrodes in alkaline solution of $1 \mathrm{M}$ ethanol and $1 \mathrm{M}$ $\mathrm{NaOH}$ show two main oxidation steps: one depicted by an anodic peak current density appearing at positive potentials $(0.032 \mathrm{~V}$, black curve, $0.056 \mathrm{~V}$, red curve and $0.054 \mathrm{~V}$, blue curve in the inset of Fig. 5) and a second step depicted by an anodic peak current density appearing at negative potentials $(-0.28 \mathrm{~V}$, black curve and $-0.26 \mathrm{~V}$, red and blue curves in Fig. 5).

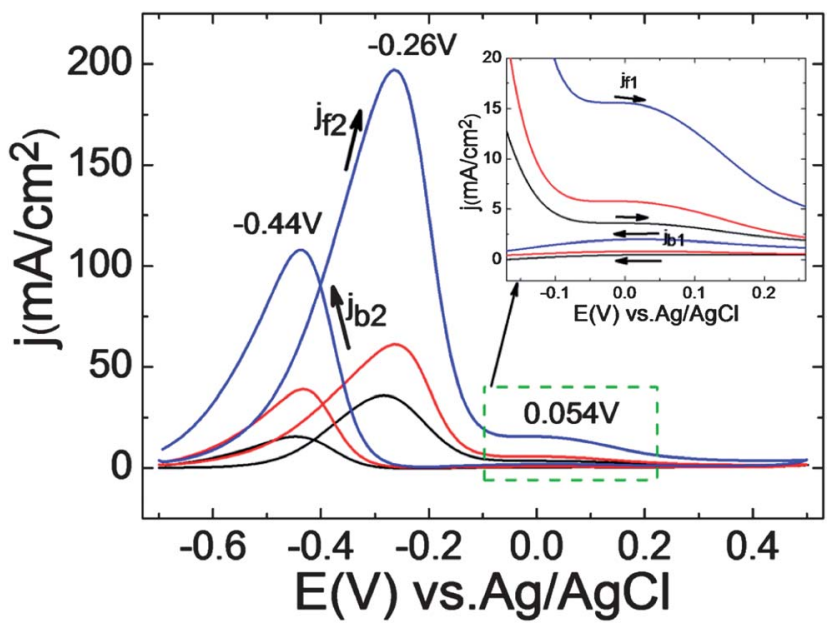

Fig. 5 Cyclic voltammograms recorded at GC-Pt1-PEI (black curve), GC-Pt2-PEI (red curve) and GC-Pt3-PEI (blue curve) modified electrodes in $1 \mathrm{M}$ ethanol and 1 $\mathrm{M} \mathrm{NaOH}$. The scan rate was $50 \mathrm{mV} \mathrm{s}^{-1}$.
Desorption of the resulting byproducts is illustrated through anodic peak current densities appearing in the reverse (backward) scan (at $-0.44 \mathrm{~V}$ for example).

The products resulting from ethanol oxidation reaction (EOR) in alkaline solution might be acetaldehyde, acetic acid or $\mathrm{CO}_{2}$ depending on the catalytic efficiency of the nanomaterials used. ${ }^{26}$ In this case, the final product of the EOR was identified to be sodium carbonate by comparison with cyclic voltammograms recorded in $0.5 \mathrm{M} \mathrm{NaOH}$ aqueous solutions of acetaldehyde, sodium acetate and purged $\mathrm{CO}_{2(\mathrm{~g})}$ (see Fig. S10†).

Acetaldehyde oxidation was identified at positive potentials $(\sim 0.054$ V, Fig. 5 and S11 (ESI $\dagger$ ) or 0.125 V in Fig. S10a $\dagger$ ) whereas no distinct oxidation current density was observed in the sodium acetate solution (red curve in Fig. S10a†). The second oxidation current density peak was attributed to $\mathrm{CO}_{3}{ }^{2-}$ resulting from the complete electro-oxidation of ethanol. The cyclic voltammograms recorded at the GC-Pt3-PEI electrode in deaerated aqueous solution of $0.5 \mathrm{M} \mathrm{NaOH}$ and purged $\mathrm{CO}_{2}$ gas show strong $\mathrm{H}_{2}$ adsorption and desorption profiles (noted with $\mathrm{H}_{\mathrm{A}}$ and $\mathrm{H}_{\mathrm{D}}$ in Fig. S10b†) in the potential range of $-0.7 \mathrm{~V}$ to -0.2 $\mathrm{V}$. The increase of the purging time of $\mathrm{CO}_{2}$ from 2 to 30 minutes, and accordingly increasing concentration of $\mathrm{CO}_{2}$ dissolved in solution, determined a shift of $\mathrm{H}_{\mathrm{A}}$ and $\mathrm{H}_{\mathrm{D}}$ peak positions toward less negative potentials. At 30 minutes purging time, the $\mathrm{H}_{2}$ desorption peaks disappear and one single oxidation peak at $-0.33 \mathrm{~V}$ starts to appear implying that the main product in the solution is $\mathrm{Na}_{2} \mathrm{CO}_{3}$ (Fig. S10b $\dagger$ ). This peak potential is very close to that obtained during ethanol oxidation at GC-Pt-PEI electrodes $(-0.28 \mathrm{~V}$ or $-0.26 \mathrm{~V}$ in Fig. 5$)$. This experimental evidence demonstrates that the ethanol electro-oxidation is complete, with $\mathrm{CO}_{2}$ being the final product (as $\mathrm{CO}_{3}{ }^{2-}$ in alkaline media), while the sensing properties of the GC-Pt3-PEI electrode for $\mathrm{CO}_{3}{ }^{2-}$ anions in solution are simultaneously revealed. Our results are consistent with the work of Datta et $a .^{\mathbf{2 6 b}}$ who identified by ion chromatography a high production of carbonate during ethanol oxidation in alkaline solution at a potential of $-0.3 \mathrm{~V}$, in the presence of $\mathrm{Pt}_{30} \mathrm{Pd}_{38} \mathrm{Au}_{32} / \mathrm{C}$ electrocatalysts.

The selectivity for the different EOR products was analyzed through the interdependence of acetaldehyde oxidation and production of $\mathrm{CO}_{2}$ at GC-Pt-PEI electrodes (Fig. S11†). For example, the first 100 consecutive CVs measured at GC-Pt2-PEI in $1 \mathrm{M}$ ethanol and $1 \mathrm{M} \mathrm{NaOH}$ aqueous solution (Fig. S11a and $\mathrm{b} \dagger$ ) show a continuous increase of oxidation peak current densities (at both positive and negative potentials).

By changing the potential window from $-0.7 \mathrm{~V}$ to $0.5 \mathrm{~V}$ (corresponding to CVs in Fig. S11a-c $\dagger$ ) to $-0.7 \mathrm{~V}$ to $0.0 \mathrm{~V}$ (orange dashed curves in Fig. S11c $\dagger$ ) and excluding the peak potential specific for acetaldehyde oxidation at $0.056 \mathrm{~V}$, one can observe a decrease of the second oxidation peak at $-0.24 \mathrm{~V}$. In other words, anything produced in step 1 is progressively consumed in step 2 . Once the production of acetaldehyde in step 1 is stopped (at $0.056 \mathrm{~V}$, Fig. S11c $\dagger$ ), the peak current density in step 2 (at $-0.24 \mathrm{~V}$, see Fig. S11c in ESI $\dagger$ ) drastically decreases because acetaldehyde (an active intermediate in this reaction) is consumed. Referring back to the scans with a full potential window $(-0.7 \mathrm{~V}$ to $0.5 \mathrm{~V})$, one can observe that the renewed 
production of acetaldehyde in step 1 determines an increase of peak current density in step 2 (compare descending orange dashed curves with ascending pink to royal curves in Fig. S11c $\dagger$ ). This is an important finding demonstrating that the $\mathrm{Pt}$ dendrites have a high selectivity for the detection of different reaction products.

Based on this experimental evidence, the overall EOR in alkaline solution at GC-Pt-PEI electrodes is:

$$
\mathrm{CH}_{3} \mathrm{CH}_{2}-\mathrm{OH}+12 \mathrm{OH}^{-} \rightarrow 2 \mathrm{CO}_{2}+9 \mathrm{H}_{2} \mathrm{O}+12 \mathrm{e}^{-}
$$

The adsorption of ethanol on the surface of the electrocatalysts as well as adsorption of $(\mathrm{OH})$ ads species responsible for further oxidation of ethanol to acetaldehyde, acetate and carbonate are well-known processes. ${ }^{26,27}$ Here, we present a qualitative assessment through voltammetric analysis of the products demonstrating that the Pt-PEI dendrites act as excellent electrocatalysts for ethanol oxidation with antifouling sensing properties.

Moreover, our results show a net influence of Pt-PEI porosity in the electrocatalytic process. The current densities in the voltammograms of ethanol oxidation increased with the porosity of the Pt dendrites (Fig. 5). Thus Pt3-PEI dendrites (Fig. 4c) generate the highest current density, $197 \mathrm{~mA} \mathrm{~cm}^{-2}$ (blue curve in Fig. 5), as compared to $61.0 \mathrm{~mA} \mathrm{~cm}^{-2}$ (Pt2-PEI, red curve - Fig. 5) and $36.0 \mathrm{~mA} \mathrm{~cm}^{-2}$ (Pt1-PEI, black curve in Fig. 5).

The maximum number of consecutive CVs recorded at the GC-Pt-PEI electrodes in $1 \mathrm{M}$ ethanol and $1 \mathrm{M} \mathrm{NaOH}$, without decrease of peak current densities, increased with Pt-PEI porosity (being $85 \mathrm{CVs}, 200 \mathrm{CVs}$ and $320 \mathrm{CVs}$ for Pt1-PEI, Pt2-PEI and Pt3-PEI, respectively), thus the Pt3-PEI catalyst showed the best cycleability performance. Furthermore, the high ratios of forward to backward anodic peak current density $\left(j_{\mathrm{f}} / j_{\mathrm{b}}\right.$ ) (see Table 1) $j_{\mathrm{f}_{1}} / j_{\mathrm{b}_{1}}=10.3, j_{\mathrm{f}_{2}} / j_{\mathrm{b}_{2}}=2.3$ (GC-Pt1-PEI), $j_{\mathrm{f}_{1}} / j_{\mathrm{b}_{1}}=5.6$, $j_{\mathrm{f}_{2}} / j_{\mathrm{b}_{2}}=1.6$ (GC-Pt2-PEI) and $j_{\mathrm{f}_{1}} / j_{\mathrm{b}_{1}}=7.8, j_{\mathrm{f}_{2}} / j_{\mathrm{b}_{2}}=2.0$ (GC-Pt3-PEI) indicate an excellent tolerance against poisoning from the byproducts resulting from the EOR. ${ }^{26 c}$ The higher the ratio of forward anodic peak current density to backward anodic peak current density, the more ethanol is oxidized to carbon dioxide. A slightly higher amount of Pt3-PEI catalysts cast on GC electrodes $(15 \mu \mathrm{L})$ allows the generation of a higher current density (up to $340 \mathrm{~mA} \mathrm{~cm}{ }^{-2}$, Fig. S13a†) and ensures prolonged reusability of the catalyst (Fig. S13a-c $\dagger$ ). TEM imaging offers another solid evidence for the reusability of the catalysts, as it reveals that the morphology of the used nanodendrites is unchanged even after a considerable number of electrocatalytic cycles, i.e. after 300 CVs (Fig. S14 $\dagger$ ).

The excellent electrocatalytic efficiency of Pt-PEI dendrites is consistent with previously published reports on different electrochemical systems showing that the electron transfer process becomes faster at electrodes modified with smaller nanomaterials but also at high surface coverage. ${ }^{28}$ The high porosity of the nanodendrites seems to have a strong influence on the electrocatalytic process. Although previous reports show that $\mathrm{Pt}$ octopods, ${ }^{29}$ nanoflowers, ${ }^{30}$ and porous nanowires ${ }^{31}$ are active electrocatalysts toward ethanol, in our case extremely high current densities $\left(36.0 \mathrm{~mA} \mathrm{~cm}{ }^{-2}, 61.0 \mathrm{~mA} \mathrm{~cm}{ }^{-2}\right.$ and $197.0 \mathrm{~mA}$ $\mathrm{cm}^{-2}$ ) were obtained at the GC-Pt-PEI modified electrodes for 1 $\mathrm{M}$ ethanol concentration, likely due to their smaller size (25 to $21 \mathrm{~nm}$ average), as well as their high porosity, surface area and affinity of the PEI polymer for diffusion processes. Current densities of $30 \mathrm{~mA} \mathrm{~cm}^{-2}, 100 \mathrm{~mA} \mathrm{~cm}{ }^{-2}, 45 \mathrm{~mA} \mathrm{~cm}{ }^{-2}$ or $25 \mathrm{~mA}$ $\mathrm{cm}^{-2}$ have been reported for EOR in alkaline solution performed at electrodes modified with large $\mathrm{Au} / \mathrm{Pt}$ bimetallic nanodendrites, ${ }^{4 a}$ small spherical $\mathrm{Pt}_{30} \mathrm{Pd}_{38} \mathrm{Au}_{32} / \mathrm{C}$ electrocatalysts, ${ }^{26 \boldsymbol{b}} \mathrm{Pt} / \mathrm{C}$ catalyst ${ }^{26 \boldsymbol{b}}$ or porous $\mathrm{Pt},{ }^{26 c}$ respectively. These reports illustrate both the size and shape effect of the tested nanomaterials for the reaction under study in alkaline solution. Also compared to those cases, our Pt-PEI dendrites show still better electrocatalytic activity for the EOR. Additionally, the PtPEI nanodendrites exhibit high selectivity for the EOR byproducts and sensitivity toward $\mathrm{CO}_{3}{ }^{2-}$ detection in solution, demonstrating a rather complex electrochemical behaviour.

\section{Conclusions}

To summarize, water-soluble monodisperse Pt nanodendrites can be obtained through the thermal reduction of $\mathrm{Pt}(\mathrm{acac})_{2}$ in DMF using PEI as the stabilizer. The particles' porosity could be easily tuned by changing the Pt precursor concentration. TEM analysis revealed that the overall nanodendrites show a multiple-crystalline nature. The Pt nanodendrites present very high catalytic activity for the ethanol oxidation reaction, which is represented by properties such as sensing, selectivity, cycleability and resistance to poisoning species. Besides, we have demonstrated that the catalytic activity increases with the porosity of the dendrites.

\section{Experimental}

\subsection{Materials}

The chemical reagents were used as received. Dimethylformamide (DMF, >98\%), branched polyethyleneimine (PEI, $M_{\mathrm{w}} \sim$ 25 000), absolute ethanol, anhydrous anisole (99.7\%), $\mathrm{HClO}_{4}$ (70\%, redistilled), bottles of $\mathrm{CO}_{2}$ gas (99\%) and sodium acetate (99\%) were provided by Sigma-Aldrich. Pt(acac) $)_{2}(98 \%)$ was obtained from Strem Chemicals Inc. $\mathrm{NaOH}$ (pellets pure) was bought from Merck and acetaldehyde (anhydrous, 99.5\%) was purchased from Fluka.

\subsection{Nanodendrites preparation}

Unless specified, all synthesis and characterization stages described in the text, took place in air. In a typical procedure, $600 \mathrm{mM}$ of PEI (concentration refers to the monomer unit, ethyleneimine) were inserted in a three-neck flask containing 30 $\mathrm{mL}$ of $\mathrm{DMF}$, followed by sonication and magnetic stirring at room temperature for $15 \mathrm{~min}$. A certain amount of $\mathrm{Pt}(\mathrm{acac})_{2}$ (varied from 3 to $30 \mathrm{mM}$ ) was then added to the above mixture. Sonication and magnetic stirring helped to form a homogeneous solution with a light yellow color. Afterwards the mixture was fastly heated to $154{ }^{\circ} \mathrm{C}$ (heating rate $\sim 15{ }^{\circ} \mathrm{C} \mathrm{min}^{-1}$ ). Within a few minutes of reflux the solution got a brown-black color and it remained at the above temperature for a designed time (1-24 $\mathrm{h})$. In certain cases an aliquot was taken from the solution after 
$\sim 20$ min of reflux to monitor the initial stages of the dendrite formation. Then the black product was precipitated by centrifuging with excess acetone (8500 rpm, 30 minutes), while another centrifugation with ethanol helped for a better washing of the nanomaterials. Finally the dendrites were redispersed in Milli-Q water. Variations of the synthetic protocol (e.g. different precursor quantities) are also described in the ESI file $\dagger$, while Table 1 in the current manuscript gives some summarized information for all samples presented in this work.

\subsection{Fabrication of platinum dendrite films for electrocatalysis}

First, glassy carbon electrodes of $0.0314 \mathrm{~cm}^{2}$ area were cleaned by polishing on microcloth pads with 0.3 and $0.05 \mu \mathrm{m}$ alumina powder and washing in Milli-Q water and ethanol by ultrasonic treatment for a few minutes. Freshly cleaned glassy carbon electrodes were modified with each individual sample of $\mathrm{Pt}$ dendrites by casting $10 \mu \mathrm{L}$ of aqueous solution $\left(2 \mathrm{mg} \mathrm{mL}^{-1}\right)$ of Pt1-PEI, Pt2-PEI or Pt3-PEI on their surface and let to dry. The Pt-PEI modified electrodes were used as working electrodes for the study of ethanol oxidation in alkaline solutions.

\subsection{Characterization}

Electron microscopy observations were carried out at the University of Antwerp, using a FEI Tecnai G2 electron microscope operated at $200 \mathrm{kV}$. A Fischione tomography holder (model 2020) was used and the series were acquired automatically using the Xplore3D software. All tilt series were acquired in HAADF-STEM mode with an angular range from $-70^{\circ}$ to $+70^{\circ}$ and a tilt increment of $2^{\circ}$. The alignment of the series was done with Inspect 3D software (FEI). 3D reconstruction was performed using the Simultaneous Iterative Reconstructive Technique (SIRT) as implemented in Inspect 3D. High resolution HAADF-STEM images were acquired using a FEI Titan 50-80 electron microscope operated at $120 \mathrm{kV}$. The conventional TEM observations were performed at the CACTI institute in Vigo using a JEOL JEM 1010 microscope operating at an acceleration voltage of $100 \mathrm{kV}$. The acquisition of X-ray diffraction patterns (XRD) necessitated the use of solid powder, which was recovered upon drying our colloidal solutions under dynamic vacuum overnight. XRD measurements were carried out at CACTI in Vigo using a Siemens D-5000 diffractometer with a

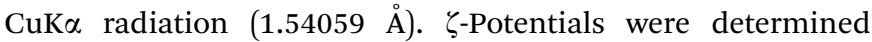
through electrophoretic mobility measurements by taking the average of five measurements at the stationary level, using a Zetasizer Nano S (Malvern Instruments) with a He-Ne laser operating at $633 \mathrm{~nm}$ and a detection angle of $173^{\circ}(4 \mathrm{~mW})$. The surface area of the dendrites was estimated at the Max-Planck Institute in Golm by BET measurements after outgassing the samples in dynamic vacuum for a few hours at moderate temperatures. The surface coverage with Pt dendrites on glassy carbon electrodes was analyzed by scanning electron microscopy (SEM) images obtained using a JEOL JSM 6700F FEG microscope operating at an acceleration voltage of $15 \mathrm{kV}$ and with a secondary-electron detector.

\subsection{Electrochemical measurements}

All electrochemical measurements were performed on a PGSTAT $302 \mathrm{~N}$ potentiostat (EcoChemie B.V., The Netherlands) in a standard three-electrode single compartment cell. Glassy carbon (GC) electrodes $\left(0.0314 \mathrm{~cm}^{2}\right.$ area) modified with Pt-PEI dendrites were used as working electrodes. The high surface coverage with Pt dendrites was confirmed by SEM analysis of GC-Pt-PEI modified electrodes (Fig. S9†). A platinum wire and an $\mathrm{Ag} / \mathrm{AgCl}(3 \mathrm{M} \mathrm{KCl})$ electrode were used as counter and reference electrodes, respectively.

The electrocatalytic behavior of Pt-PEI dendrites cast on GC electrodes was evaluated for ethanol oxidation in aqueous solution of $1 \mathrm{M}$ ethanol and $1 \mathrm{M} \mathrm{NaOH}$. For this purpose, consecutive cyclic voltammograms were recorded between the potentials $-0.7 \mathrm{~V}$ and $0.5 \mathrm{~V} v s$. $\mathrm{Ag} / \mathrm{AgCl}(3 \mathrm{M} \mathrm{KCl})$, at a scan rate of $50 \mathrm{mV} \mathrm{s}^{-1}$ up to 300 scans. The active area of the modified electrodes was evaluated by cyclic voltammetry measurements performed in $0.1 \mathrm{M} \mathrm{HClO}_{4}$ aqueous solution. Very well defined hydrogen adsorption-desorption profiles can be observed in the region of $-0.3 \mathrm{~V}$ to $0 \mathrm{~V}$ (Fig. S12†). Moreover, other assignments in the same figure include: double layer charge region ( $0 \mathrm{~V}$ to 0.2 V), typical peaks of Pt oxidation at high potentials ( $\sim 0.65$ to 0.7 $\mathrm{V}$ ) and reduction of $\mathrm{Pt}$ oxide at $0.4 \mathrm{~V}$. This represents clear evidence that the Pt dendrites were organized as dense layers on the surface of glassy carbon electrodes and that the PEI stabilizer is a very good capping agent which does not block the catalytic sites of the Pt dendrites. The CVs in Fig. 5 were normalized to the electrochemically active surface areas (EASA) of the modified electrodes. The electrochemical active areas of the modified electrodes were: $0.002 \mathrm{~cm}^{2}$ (GC-Pt1-PEI), $0.07 \mathrm{~cm}^{2}$ (GC-Pt2-PEI) and $0.0014 \mathrm{~cm}^{2}$ (GC-Pt3-PEI) respectively as determined from the cyclic voltammograms recorded in $0.1 \mathrm{M}$ $\mathrm{HClO}_{4}$ aqueous solution at the GC-Pt-PEI modified electrodes (one example is presented in Fig. S12†). These areas were estimated by integrating the cathodic current for the hydrogen adsorption reaction, correcting for the double layer charging current $^{32}$ and using a reported clean polycrystalline Pt electrode charge density of $210 \mu \mathrm{C} \mathrm{cm}{ }^{-2} \cdot{ }^{33}$ Acetaldehyde oxidation and $\mathrm{Na}_{2} \mathrm{CO}_{3}$ sensing at GC-Pt-PEI modified electrodes were also studied in alkaline solution by cyclic voltammetry measurements for the same potential window as for ethanol electrooxidation and at a scan rate of $50 \mathrm{mV} \mathrm{s}^{-1}$. Before starting each experiment, nitrogen was purged in the electrolyte solutions for 15 minutes for deaeration and continuously over the solution during measurements to prevent the interference of atmospheric oxygen. The reusability of the catalysts was confirmed by performing 3 sets of cyclic voltammetry measurements at the same GC-Pt3-PEI modified electrode in 3 freshly prepared $1 \mathrm{M}$ ethanol and $1 \mathrm{M} \mathrm{NaOH}$ aqueous solutions (Fig. S13†). The modified electrode was carefully washed with Millipore water prior to immersion into each new alkaline solution of $1 \mathrm{M}$ ethanol. The morphology of the nanodendrites was observed also after performing repeated cyclic voltammetry measurements at the GC-Pt3-PEI modified electrode in alkaline solution of $1 \mathrm{M}$ ethanol (Fig. S14 $\dagger$ ). For this purpose, the GC-Pt3-PEI modified electrode was first used for 300 consecutive scans in 1 
$\mathrm{M}$ ethanol and $1 \mathrm{M} \mathrm{NaOH}$ aqueous solution, washed with Millipore water and subsequently a formvar carbon-coated copper grid was gently pressed onto the surface of the electrode allowing the transfer of a sufficient dendrite population at the grid.

\section{Acknowledgements}

The authors would like to thank R. Rothe of the Max Planck Institute of Colloids and Interfaces in Golm for carrying out the BET surface area measurements. J. Millos is acknowledged for the XRD measurements at the CACTI institute in Vigo. We also thank E. Solla for the SEM images. M.C. gratefully acknowledges financial support from the European Union and FCT of Portugal through the fellowship SFRH/BPD/39294/2007. This research project is implemented within the framework of the Action "Supporting Postdoctoral Researchers" of the Operational Program "Education and Lifelong Learning" (Action's Beneficiary: General Secretariat for Research and Technology of Greece) and is co-financed by the European Social Fund (ESF) and the Greek State [project code PE4(1546)]. This work has also been supported by the Spanish MINECO (grant MAT201015374) and by the Xunta de Galicia/FEDER (grant 10PXIB314218PR). L.M.L.M. and S.B. acknowledge funding from the EU (ESMI, grant number FP7-INFRASTRUCT-2010-1, 262348).

\section{Notes and references}

1 (a) Z. Peng and H. Yang, Nano Today, 2009, 4, 143; (b) H. A. Gasteiger, S. S. Kocha, B. Sompalli and F. T. Wagner, Appl. Catal., B, 2005, 56, 9; (c) R. R. Barefoot and J. C. VanLoon, Anal. Chim. Acta, 1996, 334, 5; (d) W. Lu, B. X. Mi, M. C. W. Chan, Z. Hui, C. M. Che, N. Zhu and S. T. Lee, J. Am. Chem. Soc., 2004, 126, 4958.

2 (a) J. Chen, T. Herricks and Y. Xia, Angew. Chem., Int. Ed., 2005, 44, 2589; (b) L. N. Lewis, Chem. Rev., 1993, 93, 2693; (c) K. R. Williams and G. T. Burstein, Catal. Today, 1997, 38, 401.

3 (a) V. Mazumder, Y. Lee and S. Sun, Adv. Funct. Mater., 2010, 20, 1224; (b) E. Antolini and J. Perez, J. Mater. Sci., 2011, 46, 4435.

4 (a) X. Han, D. Wang, D. Liu, J. Huang and T. You, J. Colloid Interface Sci., 2012, 367, 342; (b) S. P. Jiang, Z. C. Liu and Z. Q. Tian, Adv. Mater., 2006, 18, 1068; (c) C. Bianchini and P. K. Shen, Chem. Rev., 2009, 109, 4183.

5 L. Wang and Y. Yamauchi, Chem.-Eur. J., 2011, 17, 8810.

6 (a) B. Lim and Y. Xia, Angew. Chem., Int. Ed., 2011, 50, 76; (b) L. Wang, M. Imura and Y. Yamauchi, ACS Appl. Mater. Interfaces, 2012, 4, 2865.

7 (a) E. Ramírez, L. Erades, K. Philippot, P. Lecante and B. Chaudret, Adv. Funct. Mater., 2007, 17, 2219; (b) L. M. Lacroix, C. Gatel, R. Arenal, C. García, S. Lachaize, T. Blon, B. Warot-Fonrose, E. Snoeck, B. Chaudret and G. Viau, Angew. Chem., Int. Ed., 2012, 51, 4690; (c) S. Ghosh, R. K. Sahu and C. R. Raj, J. Mater. Chem., 2011,
21, 11973; (d) J. Yin, J. Wang, M. Li, C. Jin and T. Zhang, Chem. Mater., 2012, 24, 2645.

8 M. Sanles-Sobrido, M. A. Correa-Duarte, S. Carregal-Romero, B. Rodriguez-González, R. A. Alvarez-Puebla, P. Herves and L. M. Liz-Marzán, Chem. Mater., 2009, 21, 1531.

9 (a) L. Bai, H. Zhu, J. S. Thrasher and S. C. Street, ACS Appl. Mater. Interfaces, 2009, 1, 2304; (b) P. L. Cuo, W. F. Chen, H. Y. Huang, I. C. Chang and S. A. Dai, J. Phys. Chem. B, 2006, 110, 3071; (c) H. Lee, S. E. Habas, S. Kweskin, D. Butcher, G. A. Somorjai and P. Yang, Angew. Chem., Int. Ed., 2006, 45, 7824.

10 (a) H. P. Liang, T. G. J. Jones, N. S. Lawrence, L. Jiang and J. S. J. Barnard, J. Phys. Chem. C, 2008, 112, 4327; (b) Z. Q. Tian, S. P. Jiang, Z. Liu and L. Li, Electrochem. Commun., 2007, 9, 1613.

11 S. C. Yi, C. Y. Jung and W. J. Kim, Mater. Res. Bull., 2011, 46, 2433.

12 B. Wu, N. Zheng and G. Fu, Chem. Commun., 2011, 47, 1039. 13 Z. Shen, Y. Matsuki and T. Shimoda, Chem. Commun., 2010, 46, 8606.

14 (a) C. C. Chen and P. L. Kuo, J. Colloid Interface Sci., 2006, 293, 101; (b) W. J. Song, J. Z. Du, T. M. Sun, P. Z. Zhang and J. Wang, Small, 2010, 6, 239; (c) T. Mikami, Y. Takayasu and I. Hirasawa, Chem. Eng. Res. Des., 2010, 88, 1248.

15 A. A. Ansari and J. P. Labis, J. Mater. Chem., 2012, 22, 16649. 16 (a) J. Cookson, Platinum Met. Rev., 2012, 56, 83; (b) S. W. Kim, J. Park, Y. Jang, Y. Chung, S. Hwang, T. Hyeon and Y. W. Kim, Nano Lett., 2003, 3, 1289.

17 E. Selishcheva, J. Parisi and J. Kolny-Olesiak, J. Nanopart. Res., 2012, 14, 711.

18 A. Mohanty, N. Garg and R. Jin, Angew. Chem., Int. Ed., 2010, 49, 4962.

19 Z. H. Lin, M. H. Lin and H. T. Chang, Chem.-Eur. J., 2009, 15, 4656.

20 P. C. Angelomé, H. H. Mezerji, B. Goris, I. Pastoriza-Santos, J. Pérez-Juste, S. Bals and L. M. Liz-Marzán, Chem. Mater., 2012, 24, 1393.

21 (a) B. Lim, M. Jiang, T. Yu, P. H. C. Camargo and Y. Xia, Nano Res., 2010, 3, 69; (b) M. A. Mahmoud, C. E. Tabor, M. A. ElSayed, Y. Ding and Z. L. Wang, J. Am. Chem. Soc., 2008, 130, 4590.

22 (a) L. Wang, H. Wang, Y. Nemoto and Y. Yamauchi, Chem. Mater., 2010, 22, 2835; (b) N. Ortiz and S. E. Skrabalak, Angew. Chem., Int. Ed., 2012, 51, 11757.

23 C. Wang, H. Daimon, T. Onodera, T. Koda and S. Sun, Angew. Chem., Int. Ed., 2008, 47, 3588.

24 X. Teng, X. Liang, S. Maksimuk and H. Yang, Small, 2006, 2, 249.

25 S. Cheong, J. Watt, B. Ingham, M. F. Toney and R. D. Tilley, J. Am. Chem. Soc., 2009, 131, 14590.

26 (a) W. Du, K. E. Mackenzie, D. F. Milano, N. A. Deskins, D. Su and X. Teng, ACS Catal., 2012, 2, 287; (b) J. Datta, A. Dutta and S. Mukherjee, J. Phys. Chem. C, 2011, 115, 15324; (c) M. M. Dimos and G. J. Blanchard, J. Phys. Chem. C, 2010, 114, 6019; (d) G. Cui, S. Song, P. K. Shen, A. Kowal and C. Bianchini, J. Phys. Chem. C, 2009, 113, 15639. 
27 P. A. Christensen, S. W. M. Jones and A. Hamnett, J. Phys. Chem. C, 2012, 116, 24681.

28 (a) M. Chirea, J. Borges, C. M. Pereira and A. F. Silva, J. Phys. Chem. C, 2010, 114, 9478; (b) M. Chirea, A. Cruz, C. M. Pereira and A. F. Silva, J. Phys. Chem. C, 2009, 113, 13077. 29 X. Huang, Z. Zhao, J. Fan, Y. Tan and N. Zheng, J. Am. Chem. Soc., 2011, 133, 4718.
30 L. Wei, Y. J. Fan, H. H. Wang, N. Tian, Z. Y. Zhou and S. G. Sun, Electrochim. Acta, 2012, 76, 468.

31 X. Zhang, W. Lu, J. Da, H. Wang, D. Zhao and P. A. Webley, Chem. Commun., 2009, 195.

32 E. Formo, Z. Peng, E. Lee, X. Lu, H. Yang and Y. Xia, J. Phys. Chem. C, 2008, 112, 9970.

33 J. Wang and G. M. Swain, J. Electrochem. Soc., 2003, 150, E24. 